ARTICLE

https://doi.org/10.1038/s41467-019-09812-8

\title{
Macroscale cortical organization and a default-like apex transmodal network in the marmoset monkey
}

\author{
Randy L. Buckner ${ }^{1,2,3} \&$ Daniel S. Margulies ${ }^{4}$
}

Networks of widely distributed regions populate human association cortex. One network, often called the default network, is positioned at the apex of a gradient of sequential networks that radiate outward from primary cortex. Here, extensive anatomical data made available through the Marmoset Brain Architecture Project are explored to show a homologue exists in marmoset. Results reveal that a gradient of networks extend outward from primary cortex to progressively higher-order transmodal association cortex in both frontal and temporal cortex. The apex transmodal network comprises frontopolar and rostral temporal association cortex, parahippocampal areas TH / TF, the ventral posterior midline, and lateral parietal association cortex. The positioning of this network in the gradient and its composition of areas make it a candidate homologue to the human default network. That the marmoset, a physiologically- and genetically-accessible primate, might possess a default-network-like candidate creates opportunities for study of higher cognitive and social functions.

\footnotetext{
${ }^{1}$ Department of Psychology and Center for Brain Science, Harvard University, Cambridge, MA 02138, USA. ${ }^{2}$ Department of Psychiatry, Massachusetts General Hospital, Boston, MA 02114, USA. ${ }^{3}$ Athinoula A. Martinos Center for Biomedical Imaging, Massachusetts General Hospital, Charlestown, MA 02129, USA. ${ }^{4}$ Centre National de la Recherche Scientifique, CNRS UMR 7225, Institut du Cerveau et de la Moelle épinière, Paris 75013 , France.

Correspondence and requests for materials should be addressed to R.L.B. (email: randy_buckner@harvard.edu)
} 
T he common marmoset, Callithrix jacchus, is a small New World primate that is increasingly being chosen as a model system for neuropsychiatric illness and studies of higher cognitive and social functions ${ }^{1-6}$. Of particular interest, marmosets possess a frontopolar granular area 10 sharing properties with the large-brained New World and Old World monkeys ${ }^{7-11}$ as well as the human ${ }^{12,13}$. Area 10 falls at the rostral apex of prefrontal cortex and is implicated in advanced forms of planning, abstract reasoning, and handling multiple competing task demands ${ }^{14-16}$. Area 10 , particularly its medial extent, is a consistent node in the human default network ${ }^{17}$ raising the possibility of a homologous network in the marmoset.

However, the marmoset's brain is 180th the size of the human brain. Its frontopolar region is comprised of a relatively homogenous area 10 with elements of a gradient rather than the more clearly differentiated architectonic subfields observed in the macaque and human ${ }^{8,10,12,13}$. Thus, the marmoset has intriguing relations to the human but its evolutionary distance ${ }^{18,19}$, small brain size, and less differentiated cortex raise uncertainty about how much homology should be expected.

Human association cortex is populated by a series of large-scale networks ${ }^{20,21}$. Multiple separable networks include canonical sensory-motor networks through to the widely distributed association networks. In terms of spatial topology, these networks form an orderly progression that radiates outwards from sensory cortex to transmodal association cortex ${ }^{22-24}$. A first open question is whether the marmoset possesses a macroscale organization similar to the human.

Situated at the farthest end of the macroscale sequence of networks in the human is the transmodal default network ${ }^{22,23}$. The default network behaves in peculiar ways as compared to other well-studied cortical networks. In particular, the default network increases activity when attention to the external environment is relaxed and internal, constructive modes of cognition emerge ${ }^{17,25,26}$. It is also active during directed tasks involving remembering and social inferences drawing a great deal of interes $\mathrm{t}^{27}$. A second open question is whether the marmoset possesses an apex transmodal network with homology to the human default network.

The human default network comprises widely distributed regions, including (I) medial prefrontal cortex extending from the frontal pole to the anterior cingulate, (II) precuneus, posterior cingulate and retrosplenial cortex, (III) a caudal region of the inferior parietal lobule, (IV) temporal association cortex extending into the temporal pole, and (V) parahippocampal cortex ${ }^{17,28,29}$. At a coarse scale, the five highlighted zones are repeatedly identified in analyses of human neuroimaging data and can serve as an anchor for identifying a candidate homolog, specifically the network recently labeled default network- $\mathrm{A}^{23,30}$. Default network-A is distinguished from the spatially adjacent default network-B by strong correlation with parahippocampal and retrosplenial cortex. Moreover, there is evidence in the macaque for homology 17,31-34.

A candidate homolog of default network-A is hypothesized to have three anatomical properties. First, the candidate should include regions at the transmodal ape $x^{22-24,35}$. Second, the candidate should comprise at least the five distributed zones of cortex discussed above and previously described in detail in relation to architectonic areas ${ }^{17,32}$. Some of these fields are expanded and differentiated into subfields in macaque and human, so they may also be expected to encompass a relatively smaller portion of the marmoset brain (see ref. ${ }^{36}$ ). Third, the candidate should be anatomically distinct from canonical sensory-motor hierarchies, in particular the network involving FEF and the MT complex. The reason for this third expectation is that, in the human, the two networks are functionally antagonistic $^{37,38}$. In Petrides and Pandya's ${ }^{11}$ analysis of the connectivity of the frontal pole in macaque, the absence of connectivity with regions participating in visuospatial and motor demands led to the conclusion that macaque area 10 "does not regulate attention to events occurring in external space".

In the present paper, extensive tract tracing data from the Marmoset Brain Architecture Project are combined to explore (1) whether there is a macroscale gradient of multiple networks in the marmoset that progresses from sensory zones to an apex transmodal network and (2) whether the apex transmodal network has properties to suggest provisional homology with the human default network. We discover that the marmoset possesses an apex transmodal network that has many parallels with the human default network.

\section{Results}

A macroscale gradient of sequential networks. The sequential pairings of frontal and posterior cortical injections suggest multiple distinct potentially parallel networks (Figs. 1 and 2). These multiple networks are spatially near to one another along a caudal to rostral sequence in frontal cortex.

The networks beginning with A6DR-PGM are more widespread than the lower level somatomotor networks (A4ab-A3a and $\mathrm{A} 6 \mathrm{DC}$ ) including projections from midcingulate, temporal visual and transmodal association cortex. The complexity of this distributed pattern and that of the A8aV-MT network make them difficult to sequence relative to one another. They both possess features that position them lower in the macroscale gradient of networks relative to the A47L-AIP and A10-TE3 networks. Their connections to frontopolar cortex are minimal or absent and connections to rostral temporal association cortex are also restricted. Our ordering reflects that $\mathrm{A} 8 \mathrm{aV}$ is rostral to $\mathrm{A} 6 \mathrm{DR}$.

As the sequence progresses, the network involving A47L and AIP includes extensive regions of prefrontal, temporal, and parietal association areas. The posterior projections to A47L spare MT and spread rostrally into temporal cortex. Similarly, the projections from posterior parietal cortex are broadly rostral to those involved in the A8aV-MT network, possibly capturing a feature observed in human homologs (e.g., ref. ${ }^{39}$ ).

The apex transmodal network involving A10 encompasses many zones expected of a default network candidate (see also ref. ${ }^{40}$ ). A10 receives projections from extensive regions of rostral temporal association cortex extending toward the temporal pole. Even when additional prefrontal injections were sought at or near A47L for contrast, none could be found that included comparable connections to the most rostral zones of temporal association cortex (e.g., see Case CJ800-CTBgr injection in A45 with some invasion of A47). The connections to the posterior injection (in rostral TE3) include A10 and surrounding regions, as well as prominent label in the parahippocampal region (TH/TF), modest projections from posterior cingulate/retrosplenial cortex and a zone in posterior parietal cortex on the border of $\mathrm{Opt}$, PG, and LIP.

In addition to providing evidence for a sequence of networks that situate themselves along a caudal to rostral frontal gradient, these composite patterns suggest a default-network-like candidate.

An apex transmodal network homolog of the default network. The pattern of connections to A10 is consistent with it being a component of a default network-A homolog. Figure 3 illustrates the anchor A10 case from Fig. 2 in greater detail as well as five additional cases to illustrate common patterns. The first observation is that the broad pattern is similar across several A10 injections and extends to nearby areas (A11, A9, and A32V), all 

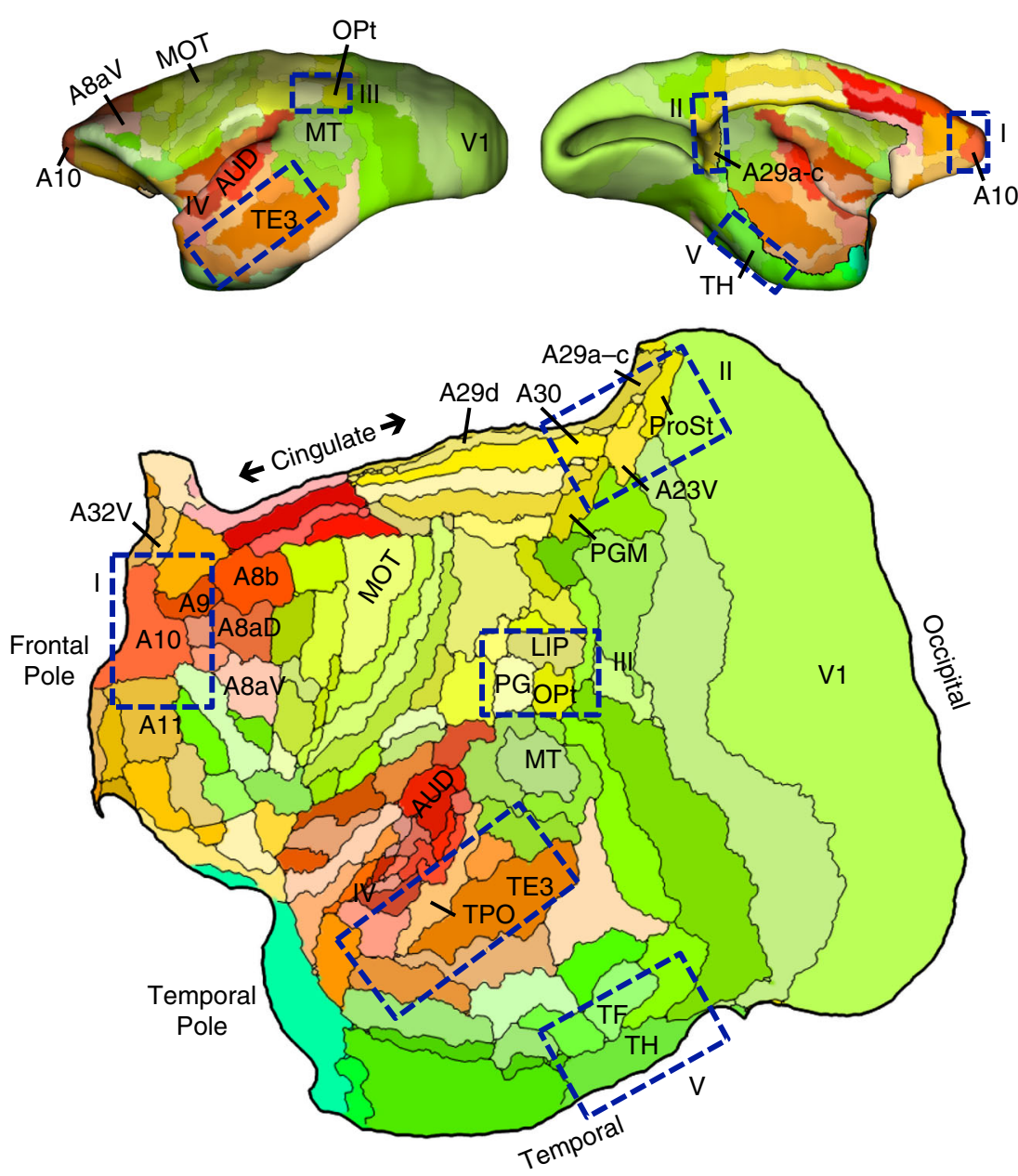

Fig. 1 Flat map format and candidate zones of interest. All cortical areas are displayed on a flat map that minimizes distortion ${ }^{58}$. The lateral (top left) and midline (top right) show the volume surface models of the marmoset cortex color-coded corresponding to the flat map representation below. Relevant areas are labeled for orientation as well as MOT (primary motor cortex A4ab) and AUD (auditory cortex involving multiple primary auditory areas). The major zones of interest in this paper are highlighted by blue rectangles: (I) frontopolar A10, (II) posterior midline A29a-c, A23, caudal A30, (III) rostral temporal association cortex TE3/TPO/PGa/IPa, (IV) posterior parietal cortex Opt/PG, and (IV) parahippocampal cortex TH/TF. These are not the only zones implicated in the default network but represent five zones that are candidate homologs to several of the well-studied regions implicated in default network-A in the human ${ }^{23}$. Area labels use nomenclature of the Paxinos et al. ${ }^{49}$ atlas

receiving projections from rostral temporal association cortex, posterior cingulate/retrosplenial cortex, and parahippocampal cortex (TH and sometimes TF). The posterior cingulate projections extend into area prostriata (ProSt) and in some cases ProSt is more densely labeled in particular near to the border of $\mathrm{A} 23 \mathrm{~V}$.

While frontopolar A10 is generally considered to be absent of direct projections from posterior parietal cortex, weak projections are present in some cases from the region of Opt/PG/LIP. When present the labeling is quite modest (e.g., Cases CJ170-DY and CJ178-CTBgr). Injection to A11, the interconnected area adjacent to A10 on the orbital surface, and A9, the area adjacent on the dorsomedial surface, show clear projections from Opt/PG/LIP while also recapitulating much of the connectional pattern of $\mathrm{A} 10$ (Cases CJ181-CTBr and CJ70-FB).

The projections to temporal association cortex provide convergent evidence with the frontopolar injections that the apex transmodal network is a default network homolog. An injection within rostral TE3 was the original target (Fig. 2). Based on its pattern, adjacent TPO was explored more thoroughly. TPO, possibly related to macaque superior temporal polysensory area defined on physiological properties ${ }^{41}$, is near to auditory cortical areas (e.g., AuCPB and AuML). This complex region thus borders higher-order auditory processing areas as well as transmodal candidates of the default network. Multiple lateral temporal lobe injections were examined that contrasted the default-networklike pattern of caudal TPO and rostral TE3 with nearby injections in auditory areas (AuCPB and AuML). The results are displayed in Fig. 4.

The TE3 injection recapitulates nearly the full extent of the candidate default network homolog (Case 180-CTBr). The caudal TPO injections (CJ122-FE and CJ122-FB) display a broad set of projections overlapping much of the zones involved in the candidate default network homolog as well as extensive local projections from auditory cortex. By contrast, the nearby injections in auditory areas AuCPB (Case CJ122-FR) and AuML (Case CJ64-FE) are predominantly local, avoiding nearly all of the default network zones. The one exception was involvement of A10 for CJ122-FR, but it should also be noted that injection, while primarily restricted to the caudal parabelt, may have involved the anterior lateral belt as well (but see also ref. ${ }^{42}$ ).

Figure $4 \mathrm{f}$ plots the locations of the temporal injections that yield the default-network-like projection pattern overlaid onto the projection patterns of the caudal-to-rostral frontal injections. The caudal TPO and rostral TE3 injections fall within or on the 


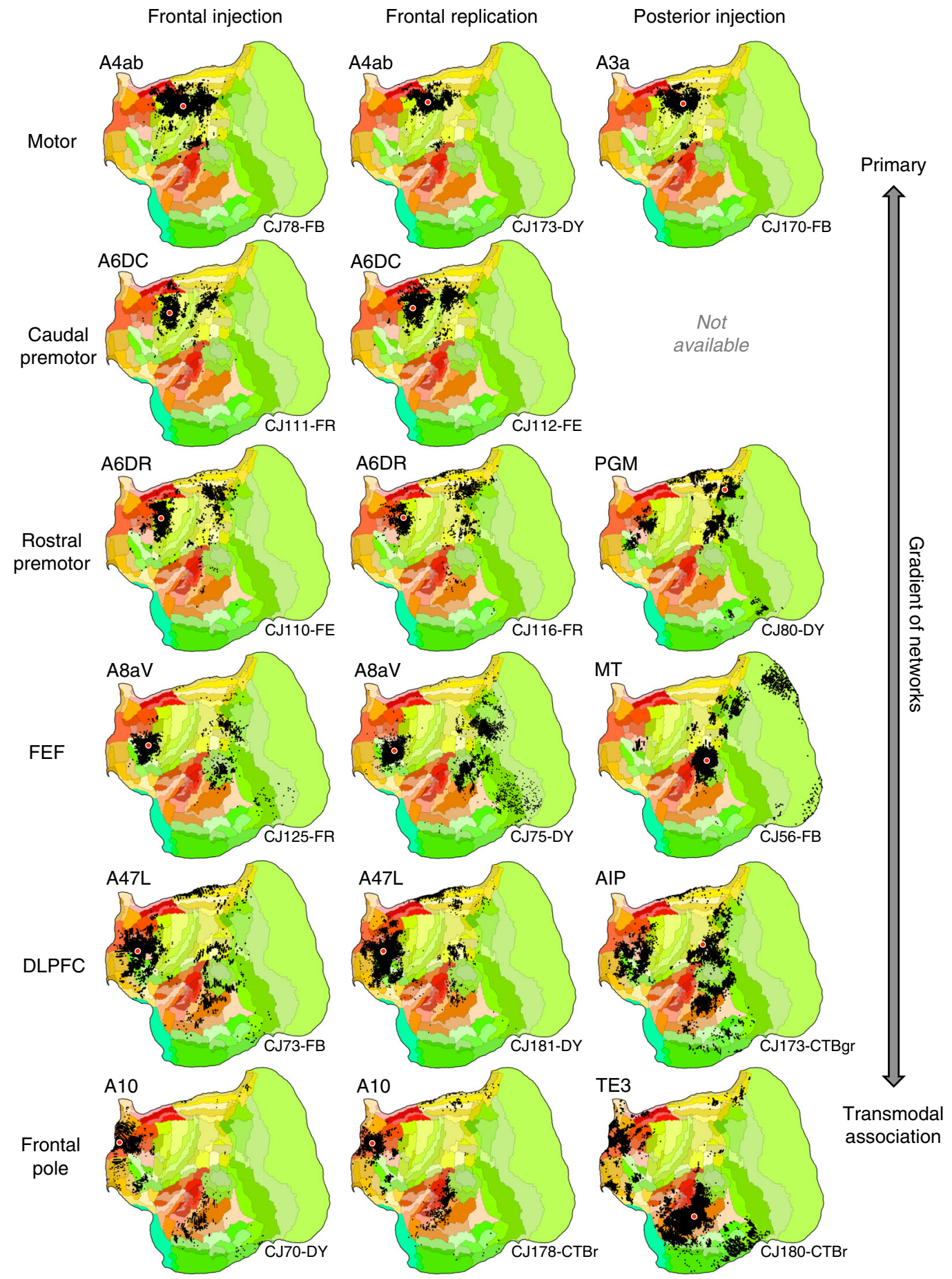

Fig. 2 Aggregate analysis of anatomical connectivity reveals a macroscale organization of networks. Each row displays a candidate network that is based on a frontal injection (left column), replication of the frontal injection (middle column), and corroboration of the network using a posterior injection (right column). Flat map format from Fig. 1. These displayed networks represent a partial subset of possible networks that could be plotted and do not reflect the full complexity of the projection patterns. Nonetheless, they reveal a sequence by which progressively more distributed networks populate the cortex as one goes from primary motor cortex (A4ab) through to frontopolar A10. In each map, the tracer injection is shown with a red dot (all retrograde). Injection cases are labeled in the bottom right as annotated in the Marmoset Brain Architecture Project archive

border of the zones linked to the apex transmodal network. The rostral TE3 injection is fully within the region of frontopolar projections, while the caudal TPO injections are on the border. Of further note is the global observation that the gradient of temporal lobe projections to frontal cortex does not respect the areal boundaries. The projection gradient goes through caudal TPO and rostral TE3, sparing large portions of the two areas.
Details of the apex transmodal network. The analyses above anchor the candidate marmoset homolog of the human default network on the inclusion of five defining zones. These zones are a subset of the complex network in the human, preferentially targeting components of what has recently been called default network- $\mathrm{A}^{23,30}$. That network shows strong coupling to the parahippocampal cortex and ventral portions of the posterior 

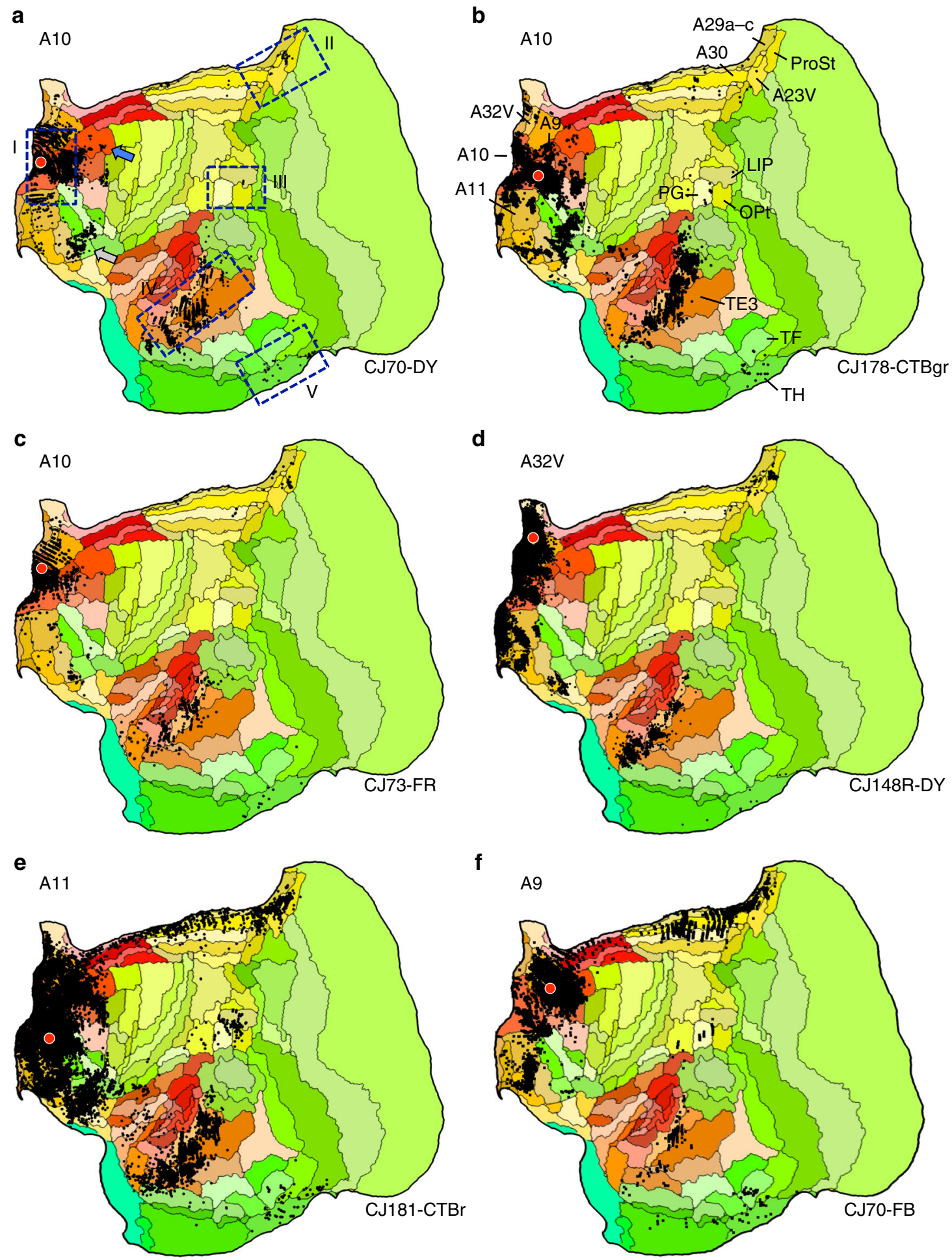

Fig. 3 Anatomical evidence for an apex transmodal network in the marmoset. a-f Multiple injections from frontopolar cortex A10 and adjacent areas are illustrated. The blue bounding boxes in a identify five target zones hypothesized to be part of the default network-A, labeled I-V. The area labels in $\mathbf{b}$ are displayed for key areas useful for orienting to components of default network-A. The blue arrow in a notes projections originating in A8ab, which is examined in more detail in Fig. 5. The gray arrow in a notes projections within and along the border of A470/A13L/A13M that are discussed in the text. Arrows also illustrate similar patterns from temporal injections in Fig. $4 \mathrm{e}$

midline and retrospenial cortex. The marmoset candidate described to this point has these properties. Motivated by recent work of Liu et al. ${ }^{43}$ focused on caudal prefrontal regions, additional tracer injections explored marmoset areas A8b (Cases CJ73-DY and CJ83-DY) and A8aD (CJ800-CTBr and CJ108-FR) in relation to the posterior midline area PGM. One injection already described in Fig. 2 was within PGM fully (CJ80-DY). An additional injection bordered A23V (Case CJ84-FB).

Figure 5 shows the results. The patterns are notable in that projections to $\mathrm{A} 8 \mathrm{~b}$ and, to a lesser extent, $\mathrm{A} 8 \mathrm{aD}$ overlap or are adjacent to zones highlighted by the analyses of the A10-TE3 network including projections from posterior cingulate, temporal 

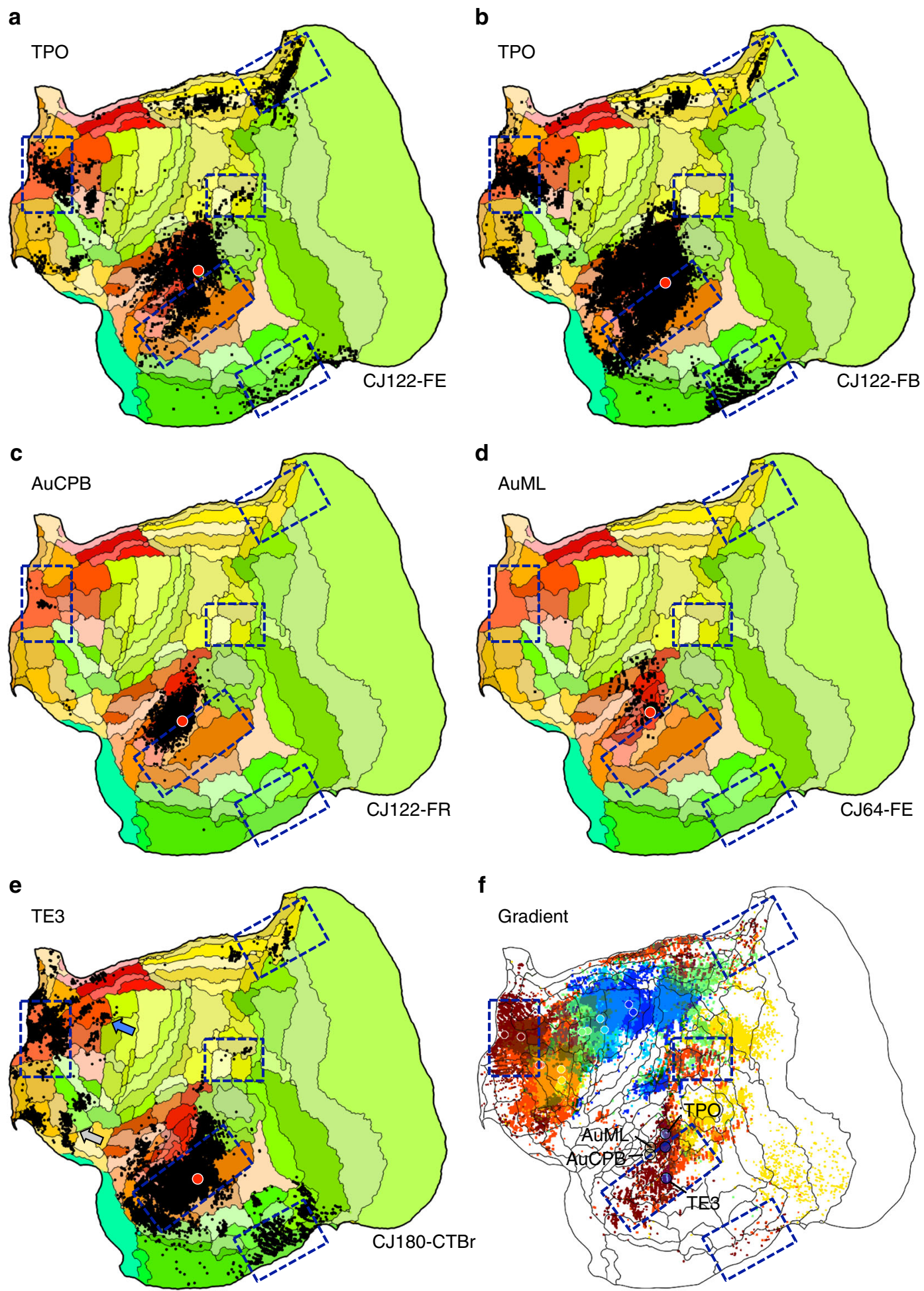

Fig. 4 Evidence for involvement of temporal association cortex in the apex transmodal network. Injections in estimated TPO (a, b) are distinct from injections to nearby auditory areas AuCPB and AuML (c, d). TPO injections receive projections from the full constellation of regions predicted as components of the default network-A candidate as well as nearby auditory areas, whereas the auditory areas receive projections predominantly from adjacent auditory areas. e Injection of TE3 also yields a default-network-like pattern, while largely sparing auditory cortex. $\mathbf{f}$ The gradient of temporal lobe projections to frontal regions is illustrated by combining the anterior projection patterns of Fig. 2 into a single multicolored image: A4ab, purple; A6DC, blue; $A 6 D R$, green; $A 8 a V$, yellow; $A 47 L$, orange; $A 10$, red. A clear progression into rostral temporal association cortex is observed. The three blue circles mark the locations of the TPO and TE3 injections and the two tan circles mark the auditory cortex injections (AuCPB and AuML). In addition to revealing the network gradient, this composite image illustrates a region of temporal cortex where transmodal association areas that are part of the default-networklike candidate are near to auditory sensory areas (see also Supplementary Fig. 1) 
a

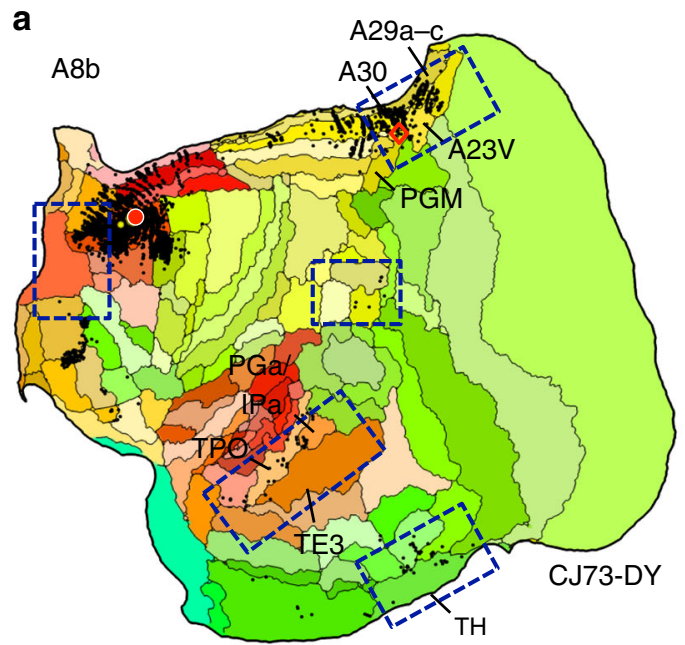

C
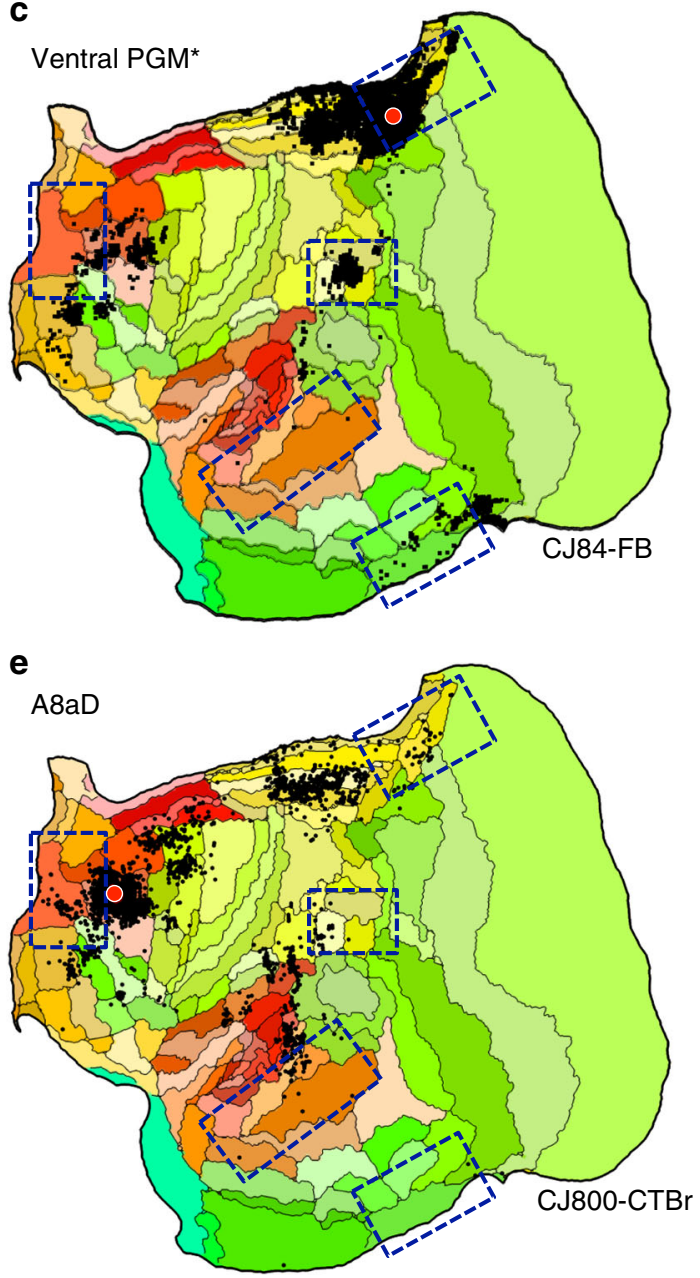

b

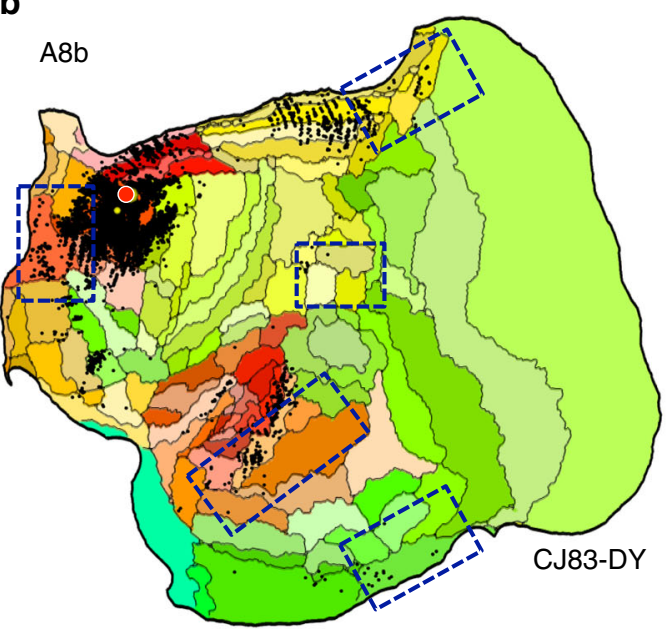

d

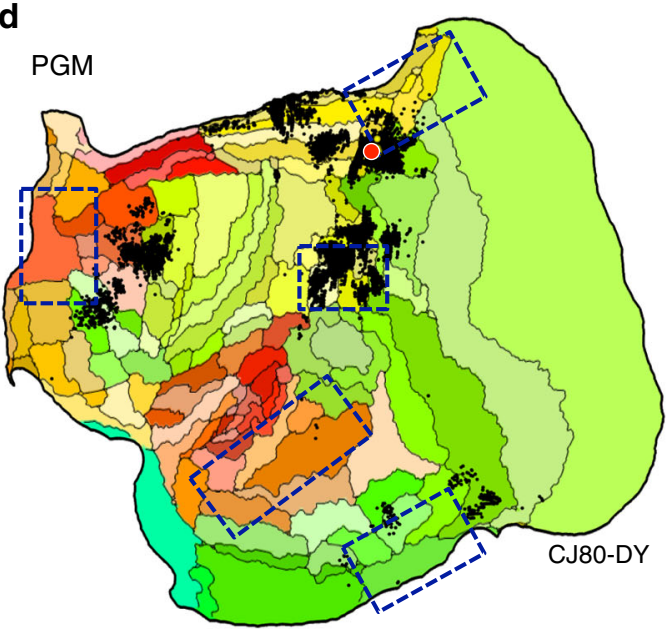

f

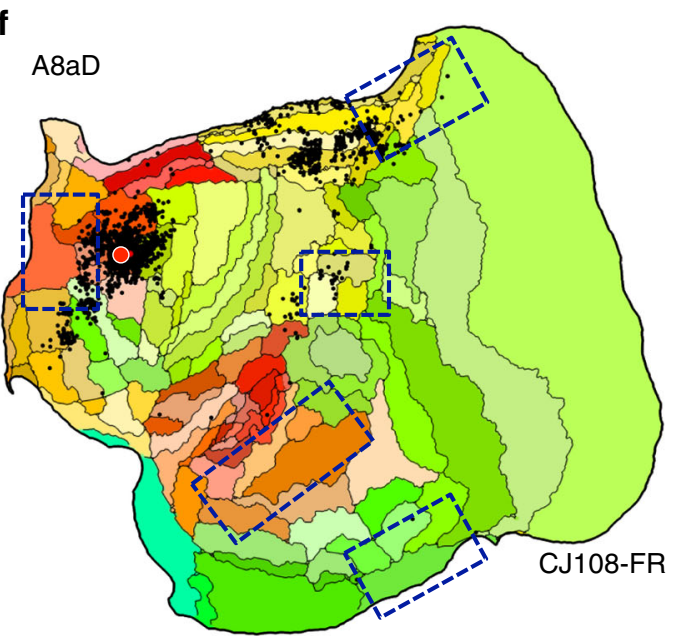

Fig. 5 Additional areas may be components or subdivisions of the apex transmodal network. The human default network contains regions outside of the five-targeted zones, including additional prefrontal regions and potentially more extensive areas along the posterior midline. Injections to two relevant frontal areas are shown, $\mathrm{A} 8 \mathrm{~b}(\mathbf{a}, \mathbf{b})$ and $\mathrm{A} 8 \mathrm{aD}(\mathbf{e}, \mathbf{f})$. The two prefrontal areas are displayed alongside posterior PGM (c, d). A8b receives projections from within or near several zones of the candidate default network, including TPO and regions along the posterior cingulate (A30, A29a-c, A23V, and ProsSt). $\mathrm{A} 8 \mathrm{~b}$ also receives projections from parahippocampal area $\mathrm{TH}$. A8aD displays overlap. PGM possesses some overlap, in particular for the injection in $\mathbf{c}$ that is in the ventral portion of PGM bordering A32V and A30. The position of the Ventral PGM injection site is shown by a red diamond in panel a to fully appreciate its location in relation to the borders of areas and in relation to the A8b injection pattern. The PGM injection falling fully within PGM reveals few (if any) projections from ventral posterior cingulate and generally spares temporal association and parahippocampal cortex 
association cortex near to $\mathrm{TPO}$ and $\mathrm{PGa} / \mathrm{IPa}$, parahippocampal cortex $\mathrm{TH} / \mathrm{TF}$, and a zone near to the Opt/PG/LIP cluster. As illustrated in Fig. 3 the A10-TE3 network receives projections from $\mathrm{A} 8 \mathrm{~b}$ and $\mathrm{A} 8 \mathrm{aD}$ (see also summary Fig. 2 of ref. ${ }^{40}$ ). The injections to PGM display less compelling involvement. In particular the injection fully in PGM has minimal projections to the posterior cingulate and retrosplenial regions implicated in the default network-A candidate. The PGM injection that shows the most integration (Case CJ84-FB) falls on the border with $\mathrm{A} 23 \mathrm{~V}$ and $\mathrm{A} 30$.

What is also revealed is that the present default-networklike candidate comprises zones beyond those typically hypothesized from the human neuroimaging literature including a single zone or multiple zones within and along the border of $\mathrm{A} 47 \mathrm{O} / \mathrm{A} 13 \mathrm{~L} / \mathrm{A} 13 \mathrm{M}$. Projections from this cluster of areas are observed for both A10 (Fig. 3a-c), TE3 (Fig. 4e), and TPO (Fig. $4 \mathrm{a}, \mathrm{b}$ ) injections. The candidate homolog of this zone is typically not well sampled in human fMRI studies because of susceptibility artifacts and therefore would not have had the opportunity to nominate itself as a consistent component of the default network (but see ref. ${ }^{44}$ ).

Taken together these additional results illustrate that the apex transmodal network is more complex than the hypothesized five zones. The caudal to rostral gradient, while capturing general features of the organization, is not a complete description of the complex topology of this network that involves multiple interdigitated areas akin to the human ${ }^{23,30}$.

A question that may arise is whether the patterns revealed above reflect unbiased properties given the several assumptions in selecting injections. To bolster confidence all of the 143 available injections were subjected to a data-driven factor analysis. Consistent with the collective results, the second major factor broadly distinguished the apex transmodal network from sensory-motor hierarchies (Supplementary Fig. 1). The one place where the distinction was not present is the region within and around auditory cortex that, as illustrated in Fig. 4, has injections that display a mixed pattern. Of interest, when the injections near to auditory cortex are analyzed together, the results reinforce separation of a preferentially auditory zone from a more rostral zone extending toward the temporal pole (beyond AuRT) that participates in the transmodal, but not auditory network (Supplementary Fig. 1 inset).

Separation from canonical sensory-motor networks. The distributed network associated with A10-TE3 is spatially separable from the A8aV-MT network across the brain (Fig. 2). To further illustrate this point, we averaged and mapped multiple projections that contrasted the two separate anterior zones (A9/A10/ $\mathrm{A} 11$ versus $\mathrm{A} 8 \mathrm{aV}$ injections) and the two separate posterior zones (TPO/TE versus MT injections). This allowed the patterns associated with the two networks to be visualized next to one another and also allowed them to be replicated from injection contrasts solely within anterior or solely within posterior cortical zones (Fig. 6). What is notable is that the candidate default network zones are distinguished from the A8aV-MT network with clear spatial separation in anterior and posterior zones of cortex, much as expected from work in the human ${ }^{37,38}$.

\section{Discussion}

The present analyses reveal that the major distributed networks that have been postulated in the human are likely conserved with the same basic organizational motif and relative positions to one another on the cortical surface of the marmoset. Many differences in detail are expected including specializations, expansions, and differences in the propensity of afferent projections

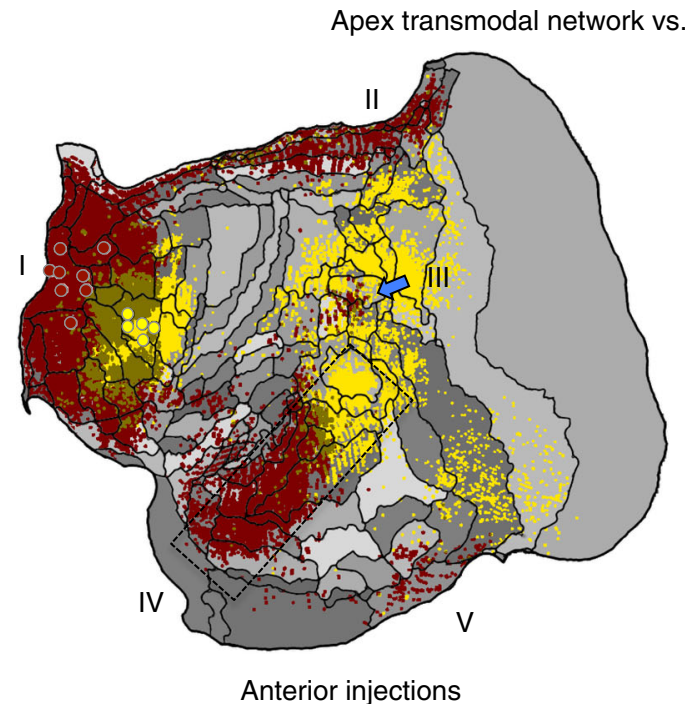

Frontopolar (A9/A10/A11) injections $(n=8)$

Candidate FEF (A8aV) injections $(n=5)$

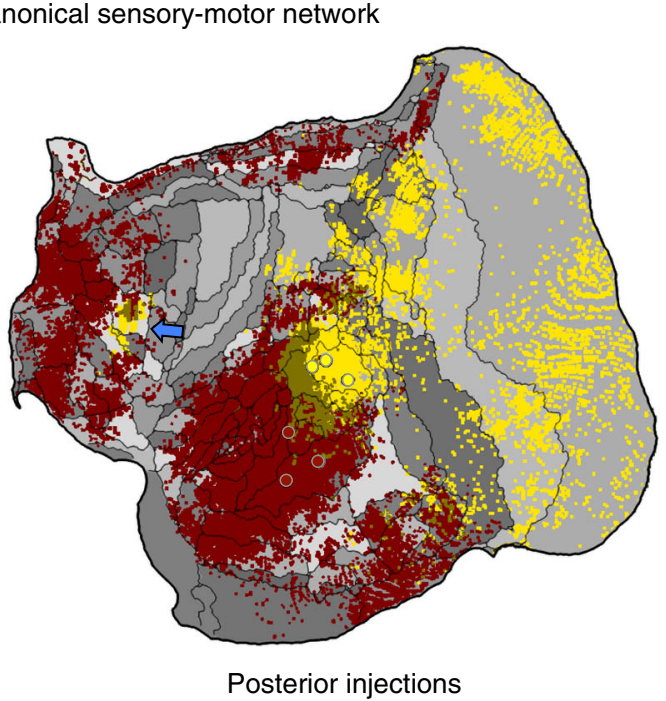

Rostral temporal (TPO/TE3) injections $(n=3)$ MT injections $(n=3)$

Fig. 6 The apex transmodal network is spatially distinct from a canonical distributed sensory-motor network. Images illustrate the contrast between injections within the apex transmodal network and the sensory-motor network linked to A8aV-MT. In each image, averages of multiple injections are contrasted. Displayed to the left are average projection maps for injections to frontopolar cortex (red) contrasted with A8aV injections (yellow). The distinct patterns of projections in posterior cortex are striking including that the apex transmodal network involves extensive regions of rostral temporal association cortex that are juxtaposed to caudal sensory-aligned regions (dashed rectangle) as well as a punctate region of parietal association cortex near $\mathrm{PG} /$ Opt that is surrounded by regions more aligned to the sensory-motor network (blue arrow). Displayed to the right are average projections for rostral temporal injections (TPO/TE3) contrasted to MT injections. This map reveals the anterior separation of the networks including the region near to A8aV (a putative homolog of FEF) that is preferentially distinct and caudal to the apex transmodal network 
to higher-order association areas but the general macroscale organization of networks is conserved including an apex transmodal network that shares homology with the human default network.

Analysis of large numbers of widely distributed tracer injections reveals a sequence of anatomical networks involving caudal to rostral frontal areas (Fig. 2). While all of the networks conform to a basic motif of anterior and posterior cortical areas, there are notable transitions between networks that might provide insight into function and how evolutionarily old motifs become expanded into the large-scale distributed networks that underpin higher cognition in primates.

In particular, the two caudalmost networks (motor: A4ab-A3a and caudal premotor: A6DC) are more locally organized than the remaining networks. Versions of this transition have been noted previously in the marmoset ${ }^{45}$ and the macaque ${ }^{46}$. In a thorough discussion of the frontoparietal network archetype, Averbeck, Caminiti and colleagues explore extensive macaque anatomical data to describe separable frontoparietal networks that have varying degrees of parallelism ${ }^{46,47}$. They observed progressively more distributed networks as the frontoparietal hierarchy was ascended with a network involving macaque PGm interconnected with a LIP/VIP/Opt cluster and a set of diverse prefrontal areas (including $8 \mathrm{a}, 8 \mathrm{~b}, 45 \mathrm{a} / \mathrm{b}$, and $46 \mathrm{v}$ ). The present transition from the caudal premotor network to the rostral premotor network, in Fig. 2, may be similar to the transition that Caminiti et al. ${ }^{46}$ describe in the macaque ${ }^{47}$ (see also ref. ${ }^{48}$ ).

Further convergent analysis comes from macaque functional connectivity data. Margulies et $\mathrm{al}^{32}$, in a comprehensive analysis of the posterior midline in macaque, noted a transition from a sensorimotor-related network to a more extensive network linked to cognitive function. Recent analyses in the marmoset using functional connectivity also reveal support for transitions from a localized somatomotor network, to a more distributed frontoparietal network candidate of the macaque FEF-MT network, and then to more rostral prefrontal networks ${ }^{34}$.

At the apex of the progression observed here in the marmoset was a transmodal network that involved frontopolar A10 and rostrotemporal TE3. The network was considerably more extensive than revealed by the initial anterior-posterior target areas, with the network including extended components of rostral prefrontal (e.g., A11, Fig. 3) and temporal association (e.g., TPO, Fig. 4) cortex, as well as areas of dorsolateral (e.g., A8b and A8aD, Fig. 5) and ventrolateral (e.g., see gray arrows in Figs. 3a and 4e) prefrontal cortex. What was striking about this network is that it mapped closely to predicted components of a homolog to the human default network.

The apex transmodal network included (I) frontopolar A10, (II) posterior midline $\mathrm{A} 29 \mathrm{a}-\mathrm{c}, \mathrm{A} 23$, rostral A30, (III) rostral temporal association cortex TE3/TPO/PGa/IPa, (IV) posterior parietal cortex Opt/PG, (IV) parahippocampal cortex $\mathrm{TH} / \mathrm{TF}$, as well as regions along the dorsolateral and ventrolateral PFC. These are the key regions expected of a homolog to default network-A. Thus the present results add support to the idea that the default network, which was discovered through functional observations in the human, arises from a network of anatomically connected regions. Moreover, the anatomically defined apex transmodal network can be distinguished from canonical sensory-motor hierarchies (Fig. 6) paralleling functional properties of the default network in the human ${ }^{37,38}$.

One particularly interesting zone emphasized in the present findings is the border of auditory cortex and the polysensory temporal area TPO. TPO injections recapitulate the complete (Fig. 4a) or nearly complete (Fig. 4b) candidate default network pattern. In addition, TPO injections label projections from auditory areas. While it is not possible with the present data to definitively interpret this juxtaposition of a default-networklike pattern and simultaneously strong projections from sensory areas, we suspect there may be distinct zones of TPO with certain regions participating in transmodel processes. The border between TPO and adjacent areas in the Paxinos et al. ${ }^{49}$ atlas has been considered for revision (ref. ${ }^{50}$ ) and the present gradient of temporal lobe projections follows a path that progresses through and divides the TPO/TE3 region. What is most interesting about this zone is the opportunity for experimental dissection.

Common marmosets are vocal primates that live in groups and co-parent providing the opportunity to study rich social behaviors $^{2}$, including productive and receptive (auditory) communication (e.g., refs. ${ }^{51-53}$ ). That so much is known about the marmoset auditory system and paradigms have been developed to task the system with simple sensory stimuli through to more elaborate social stimuli ${ }^{54,55}$ is fortuitous given that the areas involved in auditory processing are closely juxtaposed to those that are transmodal default network candidates (Fig. 4f; Supplementary Fig. 2). In future studies, the same experimental window (e.g., ref. ${ }^{56}$ ) may provide the ability to physiologically measure both auditory processing areas as well as components of the marmoset default network.

There are limitations to the approach taken here. First, in relation to the goal of drawing homologies with the human default network, the present work is based on anatomical connectivity (with reference to architectonic and positional correspondence between species). The human default network was originally defined by task suppression in neuroimaging studies and later explored in detail using functional connectivity ${ }^{17,29}$. The present analyses do not draw from physiological task suppression effects, which constitute the basis of complementary explorations ${ }^{43}$.

Another limitation is that relatively few tracer injections were available in several key zones of the apex transmodal network. Specifically, injections along the ventral posterior midline and parahippocampal cortex were absent. Only a single injection was available for rostral TE3, none for nearby temporal pole areas TE1 and TE2, and there was no posterior partner injection for A6DC (Fig. 2). There were also no injections within the unexpected projection zone along $\mathrm{A} 47 \mathrm{O} / \mathrm{A} 13 \mathrm{~L} / \mathrm{A} 13 \mathrm{M}$ to further explore this region. Greater coverage of the cortex will be critical to resolve finer details. The present results establish homology with default network-A. An open question is whether there is further organizational subdivision as recently observed in the human $^{23,30}$ or whether the marmoset network is less differentiated. Given the complexity of the network (Fig. 5) and a proposal for a default network-B candidate ${ }^{43}$, these details will be revealing.

Our analysis is also limited in its focus on retrograde tracer injections. While many projections are bidirectional, and the major networks were identified here by retrograde injections in both anterior and posterior regions, certain details are likely lost. For example, anterograde injections of the rostral temporal auditory area have demonstrated terminal label in $\mathrm{A}^{4} 0^{42}$. Similarly, our analyses focused only on monosynaptic connections. In addition to revealing second-order projections that could clarify cortical network organization, transneuronal tracer techniques will also resolve cerebrocerebellar circuit organization. The database we employed here only provided information about retrograde, monosynaptic, cortical projections.

A final limitation, perhaps to be considered a general limitation of the field, is that the areal definitions were not always predictive of the projection patterns, and in some instances, to the degree relied on as units for quantification, may prevent identifying patterns. For example, the rostral portion of TE3 received dense projections from multiple areas of the A10 network, while the 
caudal portion of the area was spared. A reasonable hypothesis is that TE3 is heterogeneous with the caudal portion more aligned to a sensory pathway and the rostral portion a transmodal zone with distinct projections (see Fig. 4f). As another example, the posterior parietal zone encompassing LIP/PG/Opt was complex with projections often on the border of the three areas. There were gradients-the A47L-AIP network projections tended to be rostral to the A8aV-MT network projections, but there was no simple alignment of projection gradients and areal boundaries, possibly reflecting a complex relationship between connectivity networks and evolutionarily new sectors of cortex (see ref. ${ }^{57}$ for discussion). Both PG and TPO in the marmoset are among the most functionally coupled areas to diverse cortical fields ${ }^{34}$ and within or near evolutionarily expanded cortical regions ${ }^{36}$.

In the future large sets of injections fully mapped to the cortical mantle may provide a means to test alternative models of network organization-models that anchor from areal definitions and boundaries, and models that examine the projection patterns in relation to gradients and interdigitated patches. The availability of large databases of mapped projections and expression patterns, in common coordinate systems and annotated by traditional architectonic areal definitions, should allow the data to reveal its organizational features. Several efforts underway are working toward such opportunities ${ }^{4,58,59}$.

Anatomical analyses of the networks that progress along a caudal to rostral gradient in the common marmoset reveal macroscale homology with the human. Just as the relative positions of primary sensory and motor areas are conserved ${ }^{60}$, it appears that the broad ordering and spatial relations between distributed association networks may also be conserved. This finding has two distinct, important implications. First, the candidate homologies provide an opportunity to study how association networks form and differentiate in ways that may go awry in neuropsychiatric and developmental stress models. That the networks share similar features to the human provide specific opportunities to explore translatable properties of higher-order association networks. Second, the homologies provide a window into the organization of association networks that evolved at least 40 millions years ago, including a candidate homolog of the default network-perhaps best to be referred to as the apex transmodal network to minimize assumptions about its functional domain. An intriguing, but unproven idea, is that the apex transmodal network may have expanded and specialized for advanced human cognitive and social capabilities. The documented homologies provide an avenue to better understand how circuits, similar to those studied extensively in the human, function and interact mechanistically using modern physiological and molecular-genetic approaches.

\section{Methods}

Overview. The present methods are based on the publically available data provided by the Marmoset Brain Architecture Project (http://www.marmosetbrain.org). This tremendously valuable open resource provides a comprehensive database with 143 retrograde tracer injections in the common marmoset (as of 8/2018) including published and unpublished injections all visualized on common flat map and surface volume projections, and annotated based on the Paxinos et al. ${ }^{49}$ atlas. Relevant methods are briefly described. The reader is referred to the original description in ref. ${ }^{58}$ for details of how the data were digitized and brought into a common atlas framework. Figure 1 illustrates the visualization format used here.

For the previously published cases, the original reports describe the projection patterns in detail ${ }^{40,61-63}$. Here, we contrast between putative separate networks and between spatially distant injections in anterior and posterior cortical zones, as described below. When injection details are described, including if an injection invaded an adjacent area, that description was derived from the annotation of the injection in the Marmoset Brain Architecture Project archive (http://www. marmosetbrain.org).

Subjects. All tracer injections were from the common marmoset (C. jacchus). Four main types of fluorescent retrograde tracers were employed as described in Majka et al. ${ }^{58}$ : flouroruby, FR; flouroemerald, FE; fast blue, FB; and diamidino yellow, DY. Several cases were not included in that original report and have subsequently been processed and uploaded to the online open resource. These cases additionally utilized tracers: choleratoxin subunit b green, CTBgr, and red, CTBr (for example, Cases CJ-CTBgr, CJ181-CTBr, and CJ180-CTBr). For the present analyses, 31 injection cases are the primary materials. Of these 12 are documented in prior publications ${ }^{40,61-63}$ and the remainder pulled from the online database ${ }^{58}$ (http:// www.marmosetbrain.org). In some instances, the maps are flipped from their original report to appear consistently in the space of the right hemisphere (e.g., Case CJ110-FE in the open release is flipped from its original presentation as Case 5 in ref. ${ }^{62}$ ). All data are readily available in the online database. Given the materials in this paper are digital projection patterns from an openly-available online database, the work is exempt from institutional ethics review.

Analyses of the gradient of networks. In order to identify candidate networks organized throughout marmoset cortex, we anchored from the hypothesis that there might be sequential networks that appear one after the other progressing from primary cortices through to higher-order transmodal association cortex ${ }^{22}$ Given many details of network organization are not known, especially for the distributed networks that populate rostral portions of frontal cortex, this endeavor must be an approximation. Networks were sought that have separable longdistance connections (e.g., to posterior cortex). Less emphasis was placed on features of local connectivity that, for example in medial and orbital frontal cortex, can include complex projections between nearby areas forming partially segregated networks ${ }^{64,65}$ (see also ref. ${ }^{40}$ ). The emphasis here is on broad macroscale organization.

To first identify possible sequential networks, frontal injection sites were selected progressing through caudal to rostral areas: (1) primary motor cortex (M1), (2) caudal premotor area A6DC, (3) rostral premotor area A6DR, (4) one of the multiple candidates for the frontal eye field (FEF) area $\mathrm{A} 8 \mathrm{aV},(5)$ prefrontal area $\mathrm{A} 47 \mathrm{~L}$, and (6) frontopolar area A10. The cascade along frontal cortex parallels the recent analyses in the human using functional neuroimaging methods ${ }^{23}$ but here operationalized in the marmoset based on anatomical tracer injections.

There were several assumptions in selecting this sequence. First, A4ab (primary motor cortex, M1) was assumed to be the lowest level. Second, A10 within the frontal pole was assumed to be at the highest transmodal level. Third, caudal premotor area A6DC was assumed to be at a relatively lower level than rostral A6DR (which directly followed M1). This relative positioning of A6DC and A6DR stems from the detailed analysis of the motor system ${ }^{45}$ where A6DC was demonstrated to have preferentially stronger projections to M1 relative to A6DR, and A6DR relatively stronger projections to posterior medial cortex (e.g., A23a-c), prefrontal, and PGM (see detailed analysis in refs. ${ }^{61,62}$ ). Recognizing that there is debate about the exact homolog(s) of FEF, A8aV was selected because of its distinct network connectivity ${ }^{7}$ and cursory parallels with network organization in the macaque $^{66,67}$ and human ${ }^{68,69}$. The placement of $\mathrm{A} 8 \mathrm{aV}$ in its specific position in the sequence is not firm; while it is assumed to come after A6DC, it is possible that it is parallel or orthogonal to A6DR. Prefrontal A47L was situated in the sequence above all of the other areas, one level below A10. A47L was selected because of availability of multiple relevant injections and because it was positioned caudal to A10 and rostral to the other selected frontal areas.

For each selected frontal injection an independent injection in another animal was identified that substantially replicated the distributed pattern of projections. The initial injection/replication pairs were as follows: A4ab (Cases CJ78-FB and CJ173-DY), A6DC (Cases CJ111-FR and CJ112-FR), A6DR (Cases CJ110-FE and CJ116-FR), A8aV (Cases CJ125-FR and CJ75-DY), A47L (Cases DJ73-FB and CJ181-DY), and A10 (Cases CJ70-DY and CJ178-CTBr).

In order to bolster confidence that the frontal injections are capturing distinct networks, the networks were all corroborated with an independent injection in one of the main posterior input areas. A4ab (primary motor cortex) was corroborated with an injection of A3a (primary somatosensory cortex; Case CJ170-FB); A6DR with an injection to medial parietal area PGM (Case CJ80-DY); A8aV with an injection to extrastriate visual area MT (CasesCJ56-FB); A47L with an injection to parietal association area AIP (Case CJ173-CTBgr), and A10 with an injection to the rostral portion of temporal association area TE3 (Case CJ180-CTBr). There was no available injection to corroborate A6DC (the only available injection in the relevant area, PE, was on the border invading somatosensory area 1/2, e.g., Case CJ173$\mathrm{CTBr}$ ). For simplicity, the network linked to each anterior-posterior injection pair is referred to by its initial targeted areas (e.g., A10-TE3 network) recognizing the full network is considerably more extensive (e.g., the A10-TE3 network likely includes A11 and TPO, among many other areas).

In depth examination of the default-network-like candidate. As the results will reveal, the marmoset possesses a set of separate networks progressing from primary motor cortex through to frontopolar cortex. The apex transmodal network associated with A10 includes many of the regions expected of a human default network homolog. To explore this network further, five additional injections were examined in and adjacent to A10. In human, the large region of medial prefrontal cortex that is involved in the default network includes area 10 (human $10 \mathrm{~m}, 10 \mathrm{r}$, and $10 \mathrm{p}$ ), area 9 , as well as anterior cingulate areas human $24 / 32$ ac (based on ref. ${ }^{13}$ as analyzed in ref. ${ }^{17}$ ). The additional injections allowed the areas in the vicinity of 
A10 to be explored more thoroughly. These injections included: caudal A10 (Case CJ178-CTBgr; with possible slight invasion of A46); medial frontopolar A10 (Case CJ73-FR), A32V (also known as 10 $\mathrm{mc}^{8}$ ); A11 (Case CJ181-CTBr with some leakage up the track in dorsolateral prefrontal cortex), and A9 (Case CJ70-FB). As the investigation progressed, additional tracer injections were examined to resolve and expand on relevant patterns.

\section{Data availability}

Data analyzed in this manuscript are available openly as part of the Marmoset Brain Architecture Project archive (http://www.marmosetbrain.org). Code used in our analyses is available at https://github.com/margulies/marmoset along with the Jupyter Notebook.

Received: 11 September 2018 Accepted: 28 March 2019

Published online: 29 April 2019

\section{References}

1. Belmonte, J. C. I. et al. Brains, genes, and primates. Neuron 86, 617-631 (2015).

2. Miller, C. T. et al. Marmosets: a neuroscientific model of human social behavior. Neuron 90, 219-233 (2016).

3. Jennings, C. et al. Opportunities and challenges in modeling human brain disorders in transgenic primates. Nat. Neurosci. 20, 1033-1033 (2017).

4. Okano, H. et al. Brain/MINDS: a Japanese national brain project for marmoset neuroscience. Neuron 92, 582-590 (2016).

5. Oikonomidis, L. et al. A dimensional approach to modeling symptoms of neuropsychiatric disorders in the marmoset monkey. Dev. Neurobiol. 77, 328-353 (2017).

6. Silva, A. C. Anatomical and functional neuroimaging in awake, behaving marmosets. Dev. Neurobiol. 77, 373-389 (2017).

7. Burman, K. J., Palmer, S. M., Gamberini, M. \& Rosa, M. G. P. Cytoarchitectonic subdivisions of the dorsolateral frontal cortex of the marmoset monkey (Callithrix jacchus), and their projections to dorsal visual areas. J. Comp. Neurol. 495, 149-172 (2006).

8. Burman, K. J. \& Rosa, M. G. P. Architectural subdivisions of medial and orbital frontal cortices in the marmoset monkey (Callithrix jacchus). J. Comp. Neurol. 514, 11-29 (2009).

9. Rosa, M. G. P. et al. Cortical afferents of area 10 in Cebus monkeys: implications for the evolution of the frontal pole. Cereb. Cortex Online. https:// doi.org/10.1091/cercor/bhy044 (2018).

10. Carmichael, S. T. \& Price, J. L. Architectonic subdivision of the orbital and medial prefrontal cortex in the macaque monkey. J. Comp. Neurol. 346, 366-402 (1994).

11. Petrides, M. \& Pandya, D. N. Efferent association pathways from the rostral prefrontal cortex in the macaque monkey. J. Neurosci. 27, 11573-11586 (2007).

12. Petrides, M. \& Pandya, D. N. Comparative architectonic analysis of the human and the macaque frontal cortex. In Handbook of Neuropsychology, Vol. 9 (eds Boller, F. \& Grafman, J.) 17-58 (Elsevier, Amsterdam, 1994).

13. Öngür, D., Ferry, A. T. \& Price, J. L. Architectonic subdivision of the human orbital and medial prefrontal cortex. J. Comp. Neurol. 460, 425-449 (2003).

14. Ramnani, N. \& Owen, A. M. Anterior prefrontal cortex: insights into function from anatomy and neuroimaging. Nat. Rev. Neurosci. 5, 184-194 (2004).

15. Tsujimoto, S., Genovesio, A. \& Wise, S. P. Frontal pole cortex: encoding ends at the end of the endbrain. Trends Cogn. Sci. 15, 169-176 (2011).

16. Mansouri, F. A., Koechlin, E., Rosa, M. G. P. \& Buckley, M. J. Managing competing goals-a key role for the frontopolar cortex. Nat. Rev. Neurosci. 18, 645-657 (2017).

17. Buckner, R. L., Andrews-Hanna, J. R. \& Schacter, D. L. The brain's default network: anatomy, function, and relevance to disease. Ann. N. Y. Acad. Sci. 1124, 1-38 (2008).

18. Perelman, P. et al. A molecular phylogeny of living primates. PLoS Genet. 7 , e1001342 (2011).

19. Hedges, S. B., Marin, J., Suleski, M., Paymer, M. \& Kumar, S. Tree of life reveals clock-like speciation and diversification. Mol. Biol. Evol. 32, 835-845 (2015).

20. Yeo, B. T. T. et al. The organization of human cerebral cortex estimated by intrinsic functional connectivity. J. Neurophysiol. 106, 1125-1165 (2011).

21. Power, J. D. et al. Functional network organization of the human brain. Neuron 72, 665-678 (2011).

22. Margulies, D. S. et al. Situating the default-mode network along a principal gradient of macroscale cortical organization. Proc. Natl Acad. Sci. USA 113, 12574-12579 (2016)
23. Braga, R. M. \& Buckner, R. L. Parallel interdigitated distributed networks within the individual estimated by intrinsic functional connectivity. Neuron 95, 457-471 (2017)

24. Huntenburg, J. M., Bazin, P. L. \& Margulies, D. S. Large-scale gradients in human cortical organization. Trends Cogn. Sci. 22, 21-31 (2018).

25. Gusnard, D. A. \& Raichle, M. E. Searching for a baseline: functional imaging and the resting human brain. Nat. Rev. Neurosci. 2, 685-694 (2001).

26. Andrews-Hanna, J. R., Smallwood, J. \& Spreng, R. N. The default network and self-generated thought: component processes, dynamic control, and clinical relevance. Ann. N. Y. Acad. Sci. 1316, 29-52 (2014).

27. Spreng, R. N., Mar, R. A. \& Kim, A. S. The common neural basis of autobiographical memory, prospection, navigation, theory of mind and the default mode: a quantitative meta-analysis. J. Cogn. Neurosci. 21, 489-510 (2009).

28. Greicius, M. D., Krasnow, B., Reiss, A. L. \& Menon, V. Functional connectivity in the resting brain: a network analysis of the default mode hypothesis. Proc. Natl Acad. Sci. USA 100, 253-258 (2003).

29. Raichle, M. E. The brain's default mode network. Annu. Rev. Neurosci. 38, 433-447 (2015).

30. Braga, R. M., van Dijk, K. R. A., Polimeni, J. R., Eldaief, M. C. \& Buckner, R. L. Parallel distributed networks resolved at high resolution reveal close juxtaposition of distinct regions. J. Neurophysiol. https://doi.org/10.1152/ jn.00808.2018 (2019).

31. Vincent, J. L. et al. Intrinsic functional architecture in the anaesthetized monkey brain. Nature 447, 83-86 (2007)

32. Margulies, D. S. et al. Precuneus shares intrinsic functional architecture in humans and monkeys. Proc. Natl Acad. Sci. USA 106, 20069-20074 (2009).

33. Binder, J. R., Desai, R. H., Graves, W. W. \& Conant, L. L. Where is the semantic system? A critical review and meta-analysis of 120 functional neuroimaging studies. Cereb. Cortex 19, 2767-2796 (2009).

34. Ghahremani, M., Hutchison, R. M., Menon, R. S. \& Everling, S. Frontoparietal functional connectivity in the common marmoset. Cereb. Cortex 27, 3890-3905 (2017).

35. Buckner, R. L. \& Krienen, F. M. The evolution of distributed association networks in the human brain. Trends Cogn. Sci. 17, 648-665 (2013).

36. Chaplin, T. A., Yu, H. H., Soares, J. G., Gattass, R. \& Rosa, M. G. P. A conserved pattern of differential expansion of cortical areas in simian primates. J. Neurosci. 33, 15120-15125 (2013).

37. Fransson, P. Spontaneous low-frequency BOLD signal fluctuations: an fMRI investigation of the resting-state default mode of brain function hypothesis. Hum. Brain Mapp. 26, 15-29 (2005).

38. Fox, M. D. et al. The human brain is intrinsically organized into dynamic, anticorrelated functional networks. Proc. Natl Acad. Sci. USA 102, 9673-9678 (2005).

39. Vincent, J. L., Kahn, I., Snyder, A. Z., Raichle, M. E. \& Buckner, R. L. Evidence for a frontoparietal control system revealed by intrinsic functional connectivity. J. Neurophysiol. 100, 3328-3342 (2008).

40. Burman, K. J., Reser, D. H., Yu, H. H. \& Rosa, M. G. P. Cortical input to the frontal pole of the marmoset monkey. Cereb. Cortex 21, 1712-1737 (2011).

41. Cusick, C. G., Seltzer, B., Cola, M. \& Griggs, E. Chemoarchitectonics and corticocortical terminations within the superior temporal sulcus of the rhesus monkey: evidence for subdivisions of superior temporal polysensory cortex. J. Comp. Neurol. 360, 513-535 (1995).

42. Reser, D. H., Burman, K. J., Richardson, K. E., Spitzer, M. W. \& Rosa, M. G. P. Connections of the marmoset rostrotemporal auditory area: express pathways for analysis of affective content in hearing. Eur. J. Neurosci. 30, 578-592 (2009).

43. Liu, C. et al. Anatomical and functional investigation of the marmoset default mode network. Nat. Commun. https://doi.org/10.1038/s41467-019-09813-7 (2019).

44. Shulman, G. L. et al. Common blood flow changes across visual tasks: II: decreases in cerebral cortex. J. Cogn. Neurosci. 9, 648-663 (1997).

45. Bakola, S., Burman, K. J. \& Rosa, M. G. P. The cortical motor system of the marmoset monkey (Callithrix jacchus). Neurosci. Res. 93, 72-81 (2015).

46. Caminiti, R., Innocenti, G. M. \& Battaglia-Mayer, A. Organization and evolution of parieto-frontal processing streams in macaque monkeys and humans. Neurosci. Biobehav. Rev. 56, 73-96 (2015).

47. Averbeck, B. B., Battaglia-Mayer, A., Guglielmo, C. \& Caminiti, R. Statistical analysis of parieto-frontal cognitive-motor networks. J. Neurophysiol. 102, 1911-1920 (2009).

48. Margulies, D. S. \& Petrides, M. Distinct parietal and temporal connectivity profiles of ventrolateral frontal areas involved in language production. J. Neurosci. 33, 16846-16852 (2013).

49. Paxinos, G., Watson, C., Petrides, M., Rosa, M. G. P., \& Tokuno, H. The Marmoset Brain in Stereotaxic Coordinates (Elsevier Academic Press, London, 2012). 
50. Majka, P. et al. Unidirectional monosynaptic connections from auditory areas to primary visual cortex in the marmoset monkey. bioRxiv, https://doi.org/ $10.1101 / 373779$ (2018)

51. Wang, X. The harmonic organization of auditory cortex. Front. Syst. Neurosci. 7, 1-11 (2013).

52. Song, X., Osmanski, M. S., Guo, Y. \& Wang, X. Complex pitch perception mechanisms are shared by humans and a New World monkey. Proc. Natl Acad. Sci. USA 113, 781-786 (2016).

53. Eliades, S. J. \& Miller, C. T. Marmoset vocal communication: behavior and neurobiology. Dev. Neurobiol. 77, 286-299 (2017).

54. Wang, X. Cortical coding of auditory features. Ann. Rev. Neurosci. 41, 527-552 (2018).

55. Nummela, S. U., Jovanovic, V., de la Mothe, L. \& Miller, C. T. Social contextdependent activity in marmoset frontal cortex populations during natural conversations. J. Neurosci. 37, 7036-7047 (2017).

56. Nishimura, M., Takemoto, M. \& Song, W.-J. Organization of auditory areas in the superior temporal gyrus of marmoset monkeys revealed by real-time optical imaging. Brain Struct. Funct. 223, 1599-1614 (2018).

57. Rosa, M. G. \& Tweedale, R. Brain maps, great and small: lessons from comparative studies of primate visual cortical organization. Philos. Trans. $R$. Soc. Lond. B Biol. Sci. 360, 665-691 (2005).

58. Majka, P. et al. Towards a comprehensive atlas of cortical connections in a primate brain: mapping tracer injection studies of the common marmoset into a reference digital template. J. Comp. Neurol. 524, 2161-2181 (2016).

59. Liu, C. et al. A digital 3D atlas of the marmoset brain based on multi-model MRI. Neuroimage 169, 106-116 (2018).

60. Krubitzer, L. The magnificent compromise: cortical field evolution in mammals. Neuron 56, 201-208 (2007).

61. Burman, K. J., Bakola, S., Richardson, K. E., Reser, D. H. \& Rosa, M. G. P. Patterns of cortical input to the primary motor area in the marmoset monkey. J. Comp. Neurol. 522, 811-843 (2014a).

62. Burman, K. J., Bakola, S., Richardson, K. E., Reser, D. H. \& Rosa, M. G. P. Patterns of afferent input to the caudal and rostral areas of the dorsal premotor cortex (6DC and 6DR) in the marmoset monkey. J. Comp. Neurol. 522, 3683-3716 (2014b).

63. Reser, D. H. et al. Contrasting patterns of cortical input to architectural subdivisions of the area 8 complex: a retrograde tracing study in marmoset monkeys. Cereb. Cortex 23, 1901-1922 (2013).

64. Carmichael, S. T. \& Price, J. L. Connectional networks within the orbital and medial prefrontal cortex of macaque monkeys. J. Comp. Neurol. 371, 179-207 (1996).

65. Öngür, D. \& Price, J. L. The organization of networks within the orbital and medial prefrontal cortex of rats, monkeys and humans. Cereb. Cortex 10, 206-219 (2000).

66. Maunsell, J. H. \& Van Essen, D. C. The connections of the middle temporal visual area (MT) and their relationship to a cortical hierarchy in the macaque monkey. J. Neurosci. 3, 2563-2586 (1983).

67. Ungerleider, L. G. \& Desimone, R. Cortical connections of visual area MT in the macaque. J. Comp. Neurol. 248, 190-222 (1986).
68. Corbetta, M. \& Shulman, G. L. Control of goal-directed and stimulus-driven attention in the brain. Nat. Rev. Neurosci. 3, 201-215 (2002).

69. Fox, M. D., Corbetta, M., Snyder, A. Z., Vincent, J. L. \& Raichle, M. E. Spontaneous neuronal activity distinguishes human dorsal and ventral attention systems. Proc. Natl Acad. Sci. USA 103, 10046-10051 (2006).

\section{Acknowledgements}

The Marmoset Brain Architecture Project (http://www.marmosetbrain.org) was the critical open resource that provided the tracer injection data. We thank Marcello Rosa and CiRong Liu for valuable discussion. Katie Insel commented on an early draft and Hannah Becker provided assistance with the references. This work was supported by NIH grant P50MH106435.

\section{Author contributions}

R.L.B. and D.S.M. conceived the experiment and analyzed the present data, and wrote the paper.

\section{Additional information}

Supplementary Information accompanies this paper at https://doi.org/10.1038/s41467019-09812-8.

Competing interests: The authors declare no competing interests.

Reprints and permission information is available online at http://npg.nature.com/ reprintsandpermissions/

Journal peer review information: Nature Communications thanks David Reser and other anonymous reviewer(s) for their contribution to the peer review of this work.

Publisher's note: Springer Nature remains neutral with regard to jurisdictional claims in published maps and institutional affiliations.

Open Access This article is licensed under a Creative Commons Attribution 4.0 International License, which permits use, sharing, adaptation, distribution and reproduction in any medium or format, as long as you give appropriate credit to the original author(s) and the source, provide a link to the Creative Commons license, and indicate if changes were made. The images or other third party material in this article are included in the article's Creative Commons license, unless indicated otherwise in a credit line to the material. If material is not included in the article's Creative Commons license and your intended use is not permitted by statutory regulation or exceeds the permitted use, you will need to obtain permission directly from the copyright holder. To view a copy of this license, visit http://creativecommons.org/ licenses/by/4.0/.

() The Author(s) 2019 